\title{
Structure of adsorbed organometallic rhodium: model single atom catalysts
}

Article

Published Version

Bennett, R. A., McCavish, N., Basham, M., Dhanak, V. and Newton, M. (2007) Structure of adsorbed organometallic rhodium: model single atom catalysts. Physical Review Letters, 98 (5). 056102. ISSN 0031-9007 doi:

https://doi.org/10.1103/PhysRevLett.98.056102 Available at https://centaur.reading.ac.uk/16789/

It is advisable to refer to the publisher's version if you intend to cite from the work. See Guidance on citing.

To link to this article DOI: http://dx.doi.org/10.1103/PhysRevLett.98.056102

Publisher: American Physical Society

All outputs in CentAUR are protected by Intellectual Property Rights law, including copyright law. Copyright and IPR is retained by the creators or other copyright holders. Terms and conditions for use of this material are defined in the End User Agreement.

\section{www.reading.ac.uk/centaur}

\section{CentAUR}

Central Archive at the University of Reading 
Reading's research outputs online 


\title{
Structure of Adsorbed Organometallic Rhodium: Model Single Atom Catalysts
}

\author{
R. A. Bennett, N. D. McCavish, and M. Basham \\ Department of Physics, University of Reading, Whiteknights, Reading, RG6 6AF, United Kingdom \\ V.R. Dhanak \\ CCLRC Synchrotron Radiation Source, Daresbury, Cheshire, WA4 2AD, United Kingdom, \\ and Physics Department, Liverpool University, Liverpool, L69 3XB, United Kingdom

\section{A. Newton} \\ The European Synchrotron Radiation Source, 6 Rue Jules Horowitz, B.P. 220, 38043 Grenoble, France
}

(Received 29 August 2006; published 2 February 2007)

\begin{abstract}
We have determined the structure of a complex rhodium carbonyl chloride $\left[\mathrm{Rh}(\mathrm{CO})_{2} \mathrm{Cl}\right]$ molecule adsorbed on the $\mathrm{TiO}_{2}(110)$ surface by the normal incidence $\mathrm{x}$-ray standing wave technique. The data show that the technique is applicable to reducible oxide systems and that the dominant adsorbed species is undissociated with $\mathrm{Rh}$ binding atop bridging oxygen and to the $\mathrm{Cl}$ found close to the fivefold coordinated Ti ions in the surface. A minority geminal dicarbonyl species, where $\mathrm{Rh}-\mathrm{Cl}$ bond scission has occurred, is found bridging the bridging oxygen ions forming a high-symmetry site.
\end{abstract}

DOI: 10.1103/PhysRevLett.98.056102

Metal-organic chemical vapor deposition (MOCVD) is the most important method employed currently for fabrication of thin metal and inorganic films on a wide range of technologically important materials, from metallic layers to III-V nitride semiconductors for optoelectronics [1]. One such area is in heterogeneous catalysis, where the ability to finely disperse reactive metal atoms is crucial to obtain the highest activities and selectivities [2]. While a great deal of research has been undertaken concerning the structure and reactivity behavior of rhodium single crystal surfaces [3], it has become clear that under real reaction conditions metal oxides and metal-organics can dominate over metallic rhodium in the desired reactive chemistry $[4,5]$. Models of fundamental catalytic processes such as $\mathrm{CO}$ oxidation and NO reduction based on metallic reactivity are potentially inappropriate when oxidic or even supported organometallic [such as $\mathrm{Rh}^{I}(\mathrm{CO})_{2}$ ] [5,6] components are present. Knowledge of the atomic scale structure of the adsorption and decomposition of metalorganic species is of fundamental importance to developing new gas sensors, catalysts, and electronic devices and in atomic layer deposition $[7,8]$.

While the normal incidence $\mathrm{x}$-ray standing wave technique (NIXSW) has been shown to be a powerful probe of adsorbate structure on metals [9], it has thus far remained absent from one of the largest and important arenas of contemporary surface science, that of the study of adsorbates on metal oxides. In fact, there are very few structural studies of complex molecular adsorbates on oxide surfaces $[10,11]$ by any technique. In this Letter, we show that NIXSW can be applied on a reducible oxide support (where bulk defects may complicate the x-ray standing wave) and can determine the surface geometric structure of a delicate organometallic molecule [12]. The NIXSW technique (comprehensively reviewed recently [13]) mod-
PACS numbers: 68.43.Fg, 68.47.Gh, 68.49.Uv, 82.33.Ya

ulates the spatial localization of a standing x-ray wave field in the crystal by scanning the photon energy through the Bragg condition which preferentially excites photoemission from surface atoms located in the antinodes. In addition to structural information, the spatial localization may be used to detail the bonding hybridization at the surface [14].

$\mathrm{TiO}_{2}(110)$ is the prototypical reducible transition metal oxide surface and is widely used as the basis for studying model catalytic systems $[15,16]$, albeit almost exclusively from vapor deposited or grown metal islands $[16,17]$. The reducibility of the substrate leads to a proliferation of bulk and surface ordered structures [18-21] as functions of stoichiometry. While structural models abound for reduced surfaces, largely based upon scanning tunneling microscopy (STM) images rather than quantitative experiments, such complexity has thwarted structural investigations of molecular adsorbates. We show that, with very careful sample preparation to maintain a reproducible bulk and surface, good structural data are forthcoming.

Rhodium was deposited by MOCVD from $\left[\mathrm{Rh}(\mathrm{CO})_{2} \mathrm{Cl}\right]_{2}$ to form an adsorbed monolayer as described previously [22]. The adsorbed $\mathrm{Rh}(\mathrm{CO})_{2} \mathrm{Cl}$ monomer is extremely sensitive to electrons and will decompose readily. Thermal decomposition (by CO desorption) also occurs slowly; however, by maintaining an overpressure of $5 \times$ $10^{-8}$ mbar CO during the experiments, the decomposition rate becomes negligible at room temperature where these experiments were conducted. The $\mathrm{Rh} 3 d, \mathrm{Cl} 2 p$, and $\mathrm{C} 1 s$ photoelectron lines give no indication of the formation of metallic $\mathrm{Rh}$ nanoparticles, confirming the retention of $\mathrm{Rh}^{I}$. The $\mathrm{TiO}_{2}$ crystals were prepared by low temperature sputter and anneal cycles to prevent formation of bulk defects (ensuring a consistent standing wave from the bulk of the crystal [23]) and became only lightly colored during ex- 
periments. NIXSW data were taken at the (110), (220), (101), and (211) Bragg reflections using photoelectron lines of highest possible sensitivity. Corrections to the dipole approximation ( $Q$ factor) were experimentally determined for the Rh and Ti $2 p$ by comparison to structural fits to Auger data (where $Q \equiv 0$ ) [24] and for the $\mathrm{C} 1 s, \mathrm{Cl}$ $1 s$, and $\mathrm{Cl} 2 p$ photoelectron lines by calculations following the literature $[25,26]$. Data reduction and line shape fitting were performed using complementary packages [27].

Figure 1(a) shows characteristic Rh NIXSW spectra and their fitting profile in three symmetrically inequivalent directions of the $\mathrm{Rh}(\mathrm{CO})_{2} \mathrm{Cl}$ covered $\mathrm{TiO}_{2}(110)$ surface. The $\mathrm{C} 1 s$ data [Fig. 1(b)] represented the most challenging problem through a very low cross section and the possibility of a large number of adsorption sites. Only the two lowest energy reflections yielded a measurable response, of which only the (110) is reliably quantifiable. Figure 1(c) shows representative Cl NIXSW photoelectron responses and fits. Clearly, the data are noisier due to the low cross section for photoemission from $\mathrm{Cl}$. The (110) response was unusually weak from the as adsorbed carbonyl chloride and displayed no enhancement in the standing wave. Table I displays the collated structural parameters derived from experiment.

The structural model built from the data was refined by minimization [28] of the distance (when plotted on Argand diagrams [29]) between experimentally determined coherent positions and fractions (Table I) and those calculated from the atomistic model, resulting from the structures reported in Table II. On the $\mathrm{TiO}_{2}(110)$ surface, the lowest symmetry site has four symmetrically equivalent positions which were all included in the fitting procedure.

The best fit in the $\mathrm{Rh}$ (110) response yields a dominant $\mathrm{Rh}$ species with $78 \%$ of the total $\mathrm{Rh}$ on the surface adsorbed atop bridging oxygen but displaced slightly towards the $(1 \overline{1} 0)$ direction. We associate this dominant species with the adsorbed carbonyl in two symmetrically equivalent sites, which have the same adsorption height with respect to the surface and hence give the uniform structure seen in STM [22]. The tilt towards the (110) direction immediately suggests that the $\mathrm{Cl}$ is associated with this species. The remaining $22 \%$ of $\mathrm{Rh}$ appears to be adsorbed bridging the bridging oxygen sites in a high-symmetry position $3.65 \AA$ above the fully coordinated $\mathrm{Ti}$ in the surface. This bridging site has been found to be preferred in $\mathrm{Rh}$ [30] and Pd [31] adsorption at low coverage in density functional theory (DFT) calculations. The ratio of 78:22 for the two adsorption sites is close to the $85: 15$ ratio derived from Fourier transform reflection-absorption infrared spectroscopy (FT-RAIRS) experiments for a geminal dicarbonyl majority species with a misoriented minority species [32].

No $\mathrm{Cl} 2 p$ NIXSW response from the (110) reflection could be observed, but the response from the (220) reflection gives a relatively high coherent fraction. The low coherent fractions in off normal directions suggest multiple adsorption sites in plane. We find that the data cannot be satisfactorily fit with two adsorption sites; three sites are the minimum required to fit the data close to within experimental error. The majority site $(61 \%)$ is in the fivefold
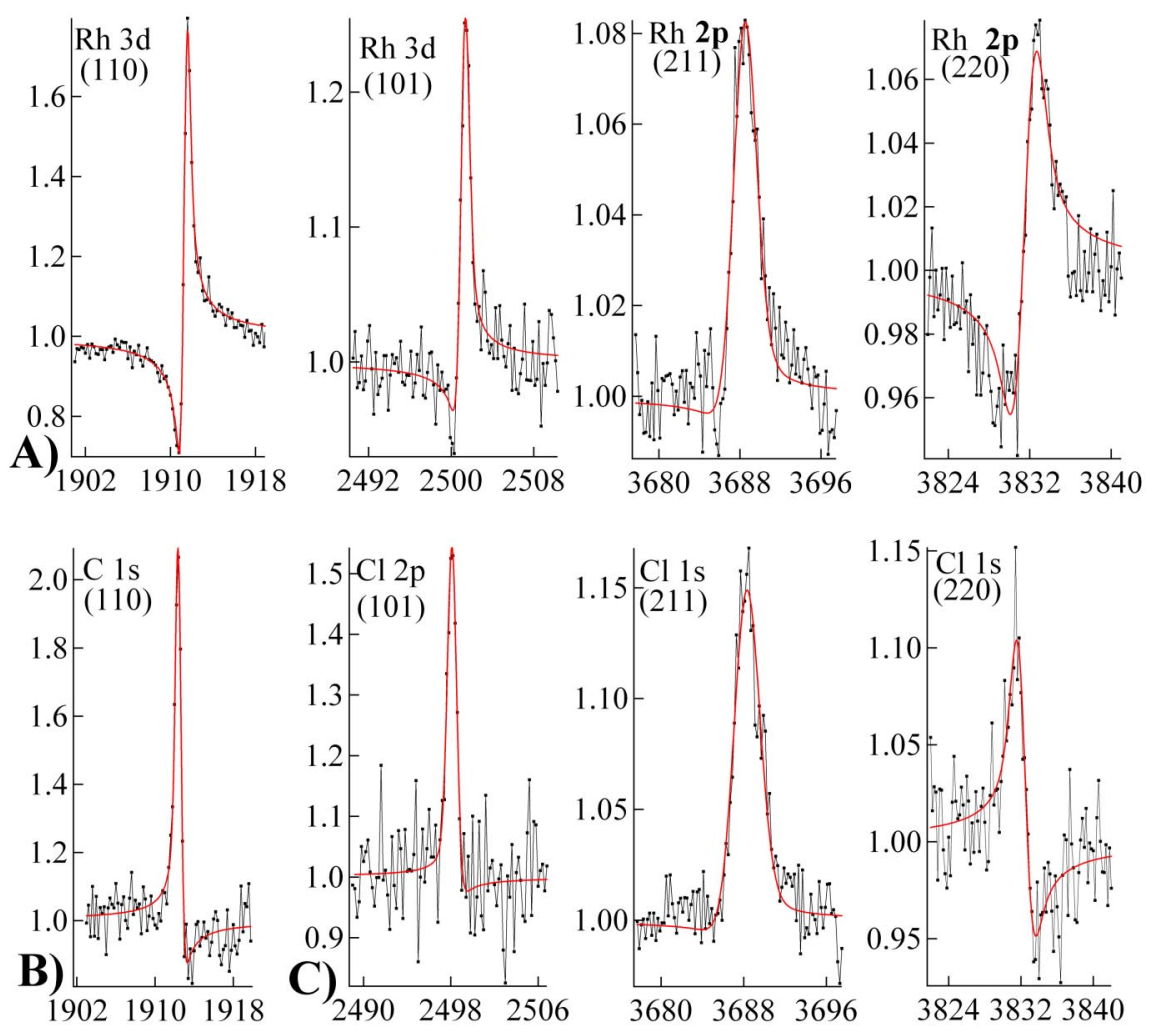

FIG. 1 (color online). (a) Rhodium, (b) carbon, and (c) chlorine NIXSW responses from as deposited saturation coverage of $\mathrm{Rh}(\mathrm{CO})_{2} \mathrm{Cl}$ under $\mathrm{CO}$. All graphs plotted normalized intensity versus photon energy in $\mathrm{eV}$. 
TABLE I. Comparison of experimentally determined coherent positions $d_{(\mathrm{hkl})}$ (in units of the interlayer spacing) and coherent fraction $f_{(\mathrm{hkl})}$ for the $\mathrm{Rh}(\mathrm{CO})_{2} \mathrm{Cl}$ adsorbed overlayer and the values predicted from the best fit structural model. The number of curves column indicates how many NIXSW fits were taken to form the mean, and the uncertainty is the standard error in the mean for multiple fits or the 1 standard deviation uncertainty returned by fitting a single curve, whichever is larger.

\begin{tabular}{ccccc}
\hline \hline Element and reflection (hkl) & No. of curves & Experiment $d_{(\mathrm{hkl})}$ & Experiment $f_{(\mathrm{hkl})}$ & Fit $d_{(\mathrm{hkl})}$ \\
\hline Rh (110) & 3 & $0.92 \pm 0.03$ & $0.77 \pm 0.02$ & 0.93 \\
Rh (101) & 4 & $0.83 \pm 0.02$ & $0.47 \pm 0.03$ & 0.88 \\
Rh (211) & 2 & $0.81 \pm 0.01$ & $0.62 \pm 0.04$ & 0.80 \\
Rh (220) & 2 & $0.82 \pm 0.01$ & $0.65 \pm 0.11$ & 0.36 \\
Cl (101) & 4 & $0.46 \pm 0.02$ & $0.32 \pm 0.03$ & 0.65 \\
Cl (211) & 3 & $0.74 \pm 0.07$ & $0.32 \pm 0.03$ & 0.46 \\
Cl (220) & 1 & $0.69 \pm 0.01$ & $0.96 \pm 0.06$ & 0.46 \\
C (110) & 3 & $0.52 \pm 0.05$ & $0.43 \pm 0.04$ & 0.69 \\
\hline \hline
\end{tabular}

coordinated Ti rows of the surface which we associate with the $\mathrm{Rh}$ majority species. This site remains virtually unchanged if the occupancy is constrained to $78 \%$ to exactly match the $\mathrm{Rh}$, which suggests a reliable determination of the site and supports the model associating majority $\mathrm{Rh}$ and $\mathrm{Cl}$ species. The $\mathrm{Cl}$ is $2.77 \AA$ above the surface Ti plane and displaced $0.66 \AA$ off the fivefold Ti sites along the (001) direction, giving a $\mathrm{Rh}-\mathrm{Cl}$ bond length of $2.38 \AA$ in comparison to $2.2 \AA$ for the free molecule. The Cl-Ti length of $2.84 \AA$ is comparable to $2.8 \AA$ found in DFT calculations for doubly coordinated $\mathrm{Cl}$ in the (001) row [33]. STM experiments [34] also show a preference for $\mathrm{Cl}$ to adsorb in the fivefold rows of the surface. The displacement from atop the fivefold $\mathrm{Ti}$ may be due to steric effects in the saturated layer.

The remaining $\mathrm{Cl}$ is distributed across two sites with optimal occupancies of $23 \%$ and $16 \%$ (15\% and $7 \%$ if the majority constraint is imposed), respectively. The structural models for the two minority species suggest adsorption on or close to the Ti fivefold site and are distinguished in adsorption height, one at $3.96 \AA$ and the other at $1.41 \AA$. These adsorption heights are nonphysical; DFT calculations find singly coordinated (atop fivefold Ti) Cl-Ti bond lengths of 2.2 and $2.33 \AA$ [33]. We consider that these adsorption sites probably represent the sum over adsorption on random surface defects such as step edges or added row strands which generate an incoherent background.
This is an important feature highlighted by Woodruff [29]; NIXSW gives an incoherent average over all regions of the surface in comparison to diffractive methods of surface structure determination, which are selectively sensitive to long range order.

In analyzing the carbon data, we draw on the developing model: Two Rh species are adsorbed at well defined occupations of $78 \%$ and $22 \%$, and reflection-absorption infrared spectroscopy (RAIRS) shows two CO molecules attached to both Rh species [32]. Symmetrical tetrahedral or nontilted square planar structures leave both $\mathrm{CO}$ molecules on each species adsorbed at the same height. The low coherent fraction in the (110) direction immediately precludes these geometries, as the data cannot be fit with the known occupations $\left(f_{(110)}\right.$ for this model is at best 0.56 in comparison to an average of $0.43 \pm 0.05$ for the experimental data). The minority $\mathrm{Rh}$ species is in a high-symmetry site, so a symmetrical structure is expected; however, the majority species is tilted towards $(1 \overline{10})$, so an asymmetric structure would be expected. We therefore use a model with three adsorption heights (two on the asymmetric majority $\mathrm{Rh}$ and one for both symmetric $\mathrm{C}$ atoms adsorbed on minority $\mathrm{Rh}$ in the $78: 22$ ratio). The majority $\mathrm{C}$ are adsorbed at 4.68 and $2.69 \AA$ above the surface, while the minority $\mathrm{C}$ atoms are $4.60 \AA$. The final proposed structure of the $\mathrm{Rh}(\mathrm{CO})_{2} \mathrm{Cl}$ species is shown in Fig. 2(a) with $\mathrm{C}-\mathrm{Rh}$ bond lengths estimated at $1.9 \AA$. The data preclude deter-

TABLE II. Fractional coverage and atomic position (in $\AA$ ) of species derived from the data. Values in brackets for $\mathrm{Cl}$ have the majority fractional coverage constrained to the majority Rh value. The origin of the coordinate system is the sixfold coordinated Ti under the bridging oxygen rows of the (110) surface, assuming it is in a bulk position. NIXSW is not sensitive to surface relaxation effects as it relies upon a bulk substrate derived x-ray standing wave.

\begin{tabular}{|c|c|c|c|c|}
\hline Element & Fractional coverage & Height in (110) $\AA$ & Displacement along (1 $1 \overline{1} 0)$ & Displacement along (001) \\
\hline Rh minority & 0.22 & 3.65 & 0.00 & 2.95 \\
\hline $\mathrm{Cl}$ majority & $0.61(0.78)$ & $2.77(2.76)$ & $3.28(3.25)$ & $0.87(0.81)$ \\
\hline $\mathrm{Cl}$ minority $\mathrm{H}$ & $0.23(0.15)$ & $3.96(3.77)$ & $3.24(3.24)$ & $1.47(1.48)$ \\
\hline $\mathrm{Cl}$ minority $\mathrm{L}$ & $0.16(0.07)$ & $1.41(1.66)$ & $3.07(3.25)$ & $1.48(1.48)$ \\
\hline $\mathrm{C}$ majority & 0.39 & 4.68 & $\ldots$ & $\ldots$ \\
\hline $\mathrm{C}$ majority & 0.39 & 2.69 & $\cdots$ & $\cdots$ \\
\hline $\mathrm{C}$ minority & 0.22 & 4.60 & $\cdots$ & $\cdots$ \\
\hline
\end{tabular}




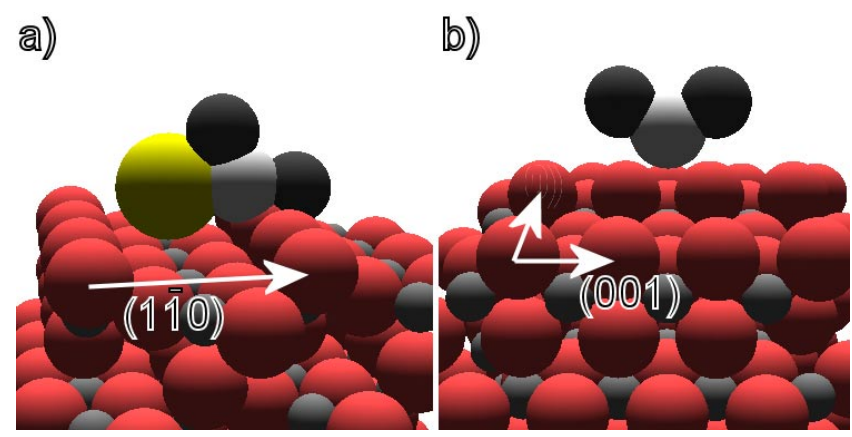

FIG. 2 (color online). (a) The proposed adsorbed local structure of the $\mathrm{Rh}(\mathrm{CO})_{2} \mathrm{Cl}$ molecule majority species with $\mathrm{Rh}$ bound to a single bridging row oxygen and (b) the minority geminal dicarbonyl species in square planar geometry bridging the bridging row oxygen. $\mathrm{C}-\mathrm{Rh}$ bond lengths are assumed to be $1.9 \AA$ and are shown in positions defined by the experimentally determined heights above the surface. Surface oxygen and titanium ions are large (red) and small (gray) spheres in the surface, respectively, while chlorine (yellow) is the largest ion; rhodium (gray) is the central ion which is bound to two (black) carbon atoms in both images. All radii are scaled to the appropriate element and ionic state; oxygen atoms in carbonyl groups are not shown for clarity.

mining the orientation of the geminal minority species; we present the preferred square planar geometry in Fig. 2(b).

In summary, we have performed extensive NIXSW measurements that show that, given careful surface preparation, the technique is employable on a weakly scattering, reducible oxide system such as $\mathrm{TiO}_{2}$, allowing determination of the adsorbed structure of a labile metal-organic compound in detail. The majority species (78\%) adsorbs in a tilted, on-top site of the bridging row oxygen ions of the surface with a lower fraction (22\%) in high-symmetry sites straddling two bridging oxygen ions. The chlorine remains associated with the majority rhodium which tilts into the row, forcing the carbonyl ligands to move out of the (110) plane. Despite the weak carbon response, symmetry considerations preclude a tetrahedral structure or a penta coordinate $\mathrm{Rh}$ as has been proposed for $\mathrm{Rh}^{I}(\mathrm{CO})_{2}$ adsorbed upon $\gamma-\mathrm{Al}_{2} \mathrm{O}_{3}$ [35]. The data in combination with previous works clearly suggest a preferred square planar geometry consistent with extended $\mathrm{x}$-ray-absorption fine structure spectroscopy results from this species formed upon $\gamma-\mathrm{Al}_{2} \mathrm{O}_{3}$ from the same precursor [36]. A geminal minority species also exists, supporting a deduction made from azimuthal angle-dependent FT-RAIRS [32].

We thank George Miller for his excellent technical assistance in setting up and running these experiments at the SRS. We thank CCLRC for beam time, the University of Reading, and the Royal Society/Wolfson Foundation for support. M. A.N. thanks the Department of Physics, University of Reading, for financial support and the ESRF for the time to make the measurements reported here.
[1] S. Nakamura, T. Mukai, and M. Senoh, Appl. Phys. Lett. 64, 1687 (1994).

[2] P. Serp and P. Kalck, Chem. Rev. 102, 3085 (2002).

[3] W. T. Wallace et al., J. Phys. Chem. B 110, 6245 (2006).

[4] M. A. Newton et al., Angew. Chem., Int. Ed. 41, 2587 (2002).

[5] M. A. Newton et al., Chem. Eur. J. 12, 1975 (2006).

[6] M. A. Newton et al., Chem. Commun. (Cambridge) 21 (2004) 2382.

[7] S. Takakusagi et al., Phys. Rev. Lett. 91, 066102 (2003).

[8] B. S. Lim, A. Rahtu, and R. G. Gordon, Nat. Mater. 2, 749 (2003).

[9] For example, A. Hauschild et al., Phys. Rev. Lett. 94, 036106 (2005); A. Gerlach et al., Phys. Rev. B 71, 205425 (2005); G. J. Jackson et al., Surf. Sci. 577, 31 (2005).

[10] Y. Iwasawa, J. Catal. 216, 165 (2003).

[11] Z. Zhang et al., Surf. Sci. 554, L95 (2004).

[12] M. A. Newton et al., Chem. Commun. (Cambridge) 17 (2000) 1677.

[13] D. P. Woodruff, Rep. Prog. Phys. 68, 743 (2005).

[14] J. C. Woicik et al., Phys. Rev. Lett. 89, 077401 (2002).

[15] R. A. Bennett et al., Mater. Sci. Technol. 18, 710 (2002).

[16] U. Diebold, Surf. Sci. Rep. 48, 53 (2003).

[17] A. Berkó, G. Ménesi, and F. Solymosi, Surf. Sci. 372, 202 (1997).

[18] R. A. Bennett et al., Phys. Rev. Lett. 82, 3831 (1999).

[19] R. A. Bennett et al., Phys. Rev. B 59, 10341 (1999).

[20] M. Blanco-Rey et al., Phys. Rev. Lett. 96, 055502 (2006).

[21] K. T. Park et al., Phys. Rev. Lett. 96, 226105 (2006).

[22] R. A. Bennett et al., Surf. Sci. 487, 223 (2001).

[23] N.D. McCavish, Ph.D. thesis, University of Reading, 2006.

[24] C. J. Fisher et al., J. Phys. Condens. Matter 10, L623 (1998).

[25] J. W. Cooper, Phys. Rev. A 47, 1841 (1993).

[26] J. J. Lee et al., Surf. Sci. 494, 166 (2001).

[27] X-ray photoemission spectroscopy quantification was undertaken in CASAXPS (http://www.casaxps.com/) and the standing wave response fit using NIXSW99\#1, R. G. Jones, Department of Chemistry, University of Nottingham, Nottingham, United Kingdom.

[28] M. Basham and R. A. Bennett, Comput. Phys. Commun. (to be published). The minimizer employed a combination of genetic algorithms and steepest descent to find global minima in structure. All quantifiable NIXSW spectra were included in the analysis for each element so that fitting automatically weighted the model towards where we had more spectra.

[29] D.P. Woodruff, B.C.C. Cowie, and A.R.H.F. Ettema, J. Phys. Condens. Matter 6, 10633 (1994).

[30] Michael Nolan, Tyndall National Institute (private communication).

[31] T. Bredow and G. Pacchioni, Surf. Sci. 426, 106 (1999).

[32] B.E. Hayden, A. King, and M. A. Newton, Chem. Phys. Lett. 269, 485 (1997).

[33] D. Vogtenhuber, R. Podloucky, and J. Redinger, Surf. Sci. 454-456, 369 (2000).

[34] U. Diebold et al., Phys. Rev. Lett. 81, 405 (1998).

[35] A. Suzuki et al., Angew. Chem., Int. Ed. 42, 4795 (2003).

[36] M. A. Newton et al., J. Phys. Chem. A 105, 5965 (2001). 